\title{
Topography-guided transepithelial accelerated corneal collagen cross-linking for low refractive error correction in keratoconus treatment: a pilot study
}

\author{
Ling Sun \\ Fudan University Eye Ear Nose and Throat Hospital Department of Ophthalmology \\ Yu Zhao \\ Fudan University Eye Ear Nose and Throat Hospital Department of Ophthalmology \\ Xiaoyu Zhang \\ Fudan University Eye Ear Nose and Throat Hospital Department of Ophthalmology \\ Mi Tian \\ Fudan University Eye Ear Nose and Throat Hospital Department of Ophthalmology \\ Jing Zhao \\ Fudan University Eye Ear Nose and Throat Hospital Department of Ophthalmology \\ Xingtao Zhou ( $\nabla$ doctzhouxingtao@163.com) \\ Fudan University Eye Ear Nose and Throat Hospital https://orcid.org/0000-0002-3465-1579
}

Research article

Keywords: pilot study, transepithelial accelerated corneal collagen cross-linking, refractive error

Posted Date: October 15th, 2019

DOI: https://doi.org/10.21203/rs.2.9443/v2

License: (a) (i) This work is licensed under a Creative Commons Attribution 4.0 International License. Read Full License 


\section{Abstract}

Purpose To investigate the safety and efficacy of topography-guided transepithelial accelerated corneal collagen cross-linking for low refractive error correction in keratoconus patients. Methods This was a prospective self-controlled study. Eighteen patients (18 eyes) were enrolled and assessed at 6 visits (pre-operation, $1 \mathrm{~d}, 1 \mathrm{~m}, 3 \mathrm{~m}, 6 \mathrm{~m}$ and 1 y post-operation). The examination at every visit included analysis of un-corrected visual acuity (UCVA), best corrected visual acuity (BCVA), corneal topography and corneal endothelial cell counts. Data are expressed as the mean \pm standard deviation (SD). The P-value was determined using repeated measures ANOVA. Results No complications occurred in any of the eyes throughout the entire follow-up period. At each visit after the operation, the corneal $\mathrm{K}$ values and spherical equivalent (SE) were reduced, while visual acuity values were increased compared with those pre-operation, although these results were not statistically significant. During the follow-up, corneal endothelial cell counts were stable. Regarding the topography, part of the corneal cone was flattened after the operation. Conclusion Topography-guided transepithelial accelerated corneal collagen cross-linking is safe and may correct low refractive error in keratoconus treatment. Further studies and improvement are needed.

\section{Introduction}

Keratoconus is a chronic, ectatic corneal disease that leads to progressive corneal thinning and irregular astigmatism. A substantial number of keratoconus patients will suffer severe visual impairment, which might severely disrupt their lives. These individuals usually use spectacles and rigid gas permeable contact lenses to improve their visual acuity, but these instruments are inconvenient and can damage the cornea when keratoconus progresses.

Currently, corneal collagen cross-linking (CXL) is accepted by ophthalmologists as a technique that both changes the biomechanics of the cornea and delays or prevents the development of corneal ectasia ${ }^{1,2}$. Meanwhile, many study results showed that patient visual acuity improved, while the corneal curvature flattened after the operation ${ }^{3-5}$. Therefore, some scientists hypothesized that corneal collagen cross-linking may be used in the treatment of low refractive error correction if this technique can be modified to obtain more obvious corneal flattening. This new technique could benefit patients who are not suited for corneal ablation. Roy et al ${ }^{6}$ found that corneal cone irradiation provided greater reductions in curvature than full corneal collagen cross-linking patterns. According to this theory, the concept of topography-guided corneal collagen cross-linking was proposed, and a related system was developed (KXL II device (Avedro Inc., Waltham, MA, USA)).

Thus far, several case reports of topography-guided transepithelial corneal collagen cross-linking have been published, and the results showed that patients demonstrated improved vision and decreased corneal curvature ${ }^{7,8}$. In this prospective study, we investigated the feasibility of topography-guided transepithelial accelerated corneal collagen cross-linking for low refractive error correction in keratoconus patients.

\section{Materials And Methods}

\section{Subjects}

This was a prospective self-controlled study. Eighteen patients (18 eyes) were recruited in this study from the Department of Ophthalmology of Fudan University EENT Hospital. The eyes enrolled were diagnosed with keratoconus and kept stable for 12 months. Stability of keratoconus was defined as less than 0.5 diopter(D) increase in maximum keratometry, manifest cylinder, or manifest refraction spherical equivalent, or no loss or loss of one line of corrected distance visual acuity over the previous 12 months. The diagnosis of keratoconus was based on corneal topography data and stromal thinning.

Inclusion criteria were the following: patients aged at least 18 years ;

CDVA of 20/80 or better; SE 0 to -3.00 D; minimal corneal thickness 475 micron or better; clear cornea without visible scar on slit-lamp examination.

Exclusion criteria were the following: previous ocular trauma or surgery; other ocular disease or systemic disease that may affect the cornea; taking Vitamin C (ascorbic acid) supplements within 1 week of the cross-linking treatment.

The Amsler-Krumeich (AK) classification of keratoconus was used in this study. Mean central $\mathrm{K}$ readings of less than 48.0 diopters (D) in addition to eccentric steepening and mild myopia and astigmatism indicate stage 1 disease. Stage II disease has mean central $\mathrm{K}$ 
readings less than 53.00 D and increasing myopia and astigmatism with the absence of scarring. Stage III disease has mean central $\mathrm{K}$ readings of greater than $53.00 \mathrm{D}$, myopia, and astigmatism from 8.00 to $10.00 \mathrm{D}$ and minimum corneal thickness of 300 to $400 \mathrm{~mm}$. Finally, stage IV disease is indicated by central corneal scarring with mean central $\mathrm{K}$ readings greater than $55.00 \mathrm{D}$ with immeasurable refractions.

Routine preoperative examinations were performed to exclude patients with contraindications. All patients fully understood the procedure and signed informed consent. This study was approved by the Ethical Committee of Fudan University EENT Hospital Review Board and followed the tenets of the Helsinki declaration.

In each visit, the following parameters were assessed: corrected distance visual acuity (CDVA), slit- lamp biomicroscopy, corneal topography, optical tomography and pachymetry with the Pentacam (Oculus, Germany) and endothelial biomicroscopy (NIDEK, Japan). All post- operative complications were recorded.

Surgical techniques

All operations were performed by the same surgeon (ZXT). After topical anesthesia with $4 \%$ oxybuprocaine, $0.1 \%$ riboflavin (ParaCel, Avedro Inc.) was applied to the cornea for 4 minutes, after which $0.25 \%$ riboflavin (VibeX xtra, Avedro Inc.) was applied for 6 minutes. The cornea was irradiated with UVA light at $45 \mathrm{~mW} / \mathrm{cm}^{2}$ and pulsed illuminated. Total UVA dose was $5.4 \mathrm{~J}$ when the corneal curvature was no more than 48D, 10J when it was 48D-51D, and 15J when it was larger than 51D. The irradiation time and shape were calculated by the KXL II system (Avedro Inc., USA) using corneal topography data(Fig.1). At the end of operation, a bandage contact lens (ACUVE OASYS, Johnson \& Johnson Inc.) was placed over the cornea.

Post-operative medication

The post-operative topical medication regimens were identical for each eye: $0.3 \%$ levofloxacin 4 times per day for 3 days, a $0.1 \%$ fluorometholone solution tapered from 6 times per day to 1 time per day over the course of 18 days, and a preservative-free tear supplement 4 times per day for 1 month. The contact lens was removed on day 1 post-operation.

Follow-up

The patients were followed up at 1 day, 1 month, 3 months, 6 months and 1 year post-operatively. The examinations included UDVA, CDVA, endothelial cell density and Pentacam corneal topography to get values of flat $\mathrm{K}(\mathrm{K} 1)$, steep $\mathrm{K}(\mathrm{K} 2)$, mean $\mathrm{K}(\mathrm{Km})$, and maximum $\mathrm{K}$ (Kmax).

Statistical analysis

Data are expressed as the mean \pm standard deviation (SD). The P-value was determined using single-factor repeated measures ANOVA. A P value $<0.05$ was considered statistically significant. All statistical analyses were performed using SPSS software (version 16.0, SPSS Inc., Chicago, IL).

\section{Results}

No complications occurred in any of the 18 patients (18 eyes). After the operation, one patient withdrew due to a personal reason. Seventeen patients (17 eyes) were enrolled for data analyses. The patients' mean age was $24.88 \pm 4.64$ years, and the male to female ratio was 9:8. One patient was lost after the 6-month follow-up.

The spherical equivalent (SE), uncorrected distant visual acuity (UDVA), and corrected distant visual acuity (CDVA) results at each followup visit are shown in Table 1. After the operation, the mean SE of each follow-up point decreased compared to that pre-operation. At the 1-year post-operation visit, the average SE decreased by $0.73 \mathrm{D}$ compared to the pre-operation SE. This finding was not statistically significant $(P>0.05)$. The post-operation UDVA and CDVA were both increased, although the differences were not significant $(P>0.05)$. The UCVA of most patients was higher than the pre-operative value, and the UCVA of nearly $1 / 3$ of the patients was enhanced by at least 3 lines at each follow-up visit(Fig.2). At the last visit, the mean postoperative CDVA and the mean postoperative UCVA were $0.05 \pm 0.04$ and $0.52 \pm 0.08$. The safety index (postoperative CDVA/ preoperative CDVA) and the efficacy index(postoperative UCVA/ preoperative CDVA) were $0.56 \pm 1.0$ and $5.78 \pm 2.0$.

The results of corneal topography examination at each follow-up visit are shown in Table 1. The average post-operative flat $\mathrm{K}$ value (K1), steep K value (K2), mean K value (Km), and maximum K value (Kmax) decreased compared with those determined preoperatively; 
however, this finding was not statistically significant $(P>0.05)$. The corneal curvatures changed approximately $0.5 \mathrm{D}$, while the Kmax of 2 eyes decreased 1.5D. Some corneal cones demonstrated flattening in the topography image during the whole follow-up (Fig. 3).

During the follow-up, corneal endothelial cell counts kept stable(Table 1).

\section{Discussion}

Topography-guided corneal collagen cross-linking is a new technique that has been developed for refractive error correction, which efficacy may still be limited; however, to keratoconus patients, the technique is meaningful since it can not only correct refractive error to some extent but also strengthen the cornea and delay or prevent disease progression. Our study was a prospective self-controlled study and investigated the safety and efficacy of topography-guided transepithelial accelerated corneal collagen cross-linking for low refractive error correction in keratoconus treatment.

All operations were uneventful, and no corneal infection, haze or other complications had occurred by the 1-year follow-up. Only moderate corneal epithelial edema was found on the first day after operation. At the 1-month post-operative visit, all corneal epithelia recovered completely. The corneal endothelial cell counts did not change significantly at each follow-up visit. These results were similar to those achieved with transepithelial accelerated cross-linking using the same UVA power ${ }^{9}$, which indicated that topography-guided transepithelial accelerated corneal collagen cross-linking may have a good safety comparable to that of general transepithelial accelerated corneal cross-linking.

At the 1-year post-operation visit, the average SE was decreased by $0.73 \mathrm{D}$, while it declined by more than $1 \mathrm{D}$ in 6 eyes (35.3\%). The maximum reduction was 1.75D. In 9 patients, UDVA was enhanced by 2 lines or more. Since an improvement of more than 1D of refraction and 2 lines of vision can be considered clinically significant, these results suggested that topography-guided transepithelial accelerated corneal collagen cross-linking may correct a low degree of refractive error correction in some patients.

The Kmax in 2 eyes decreased by 1.5D at the 3-month post-operation visit, while the other eyes' Kmax value changed by approximately $0.5 \mathrm{D}$ throughout the entire follow-up period. The values of $\mathrm{K} 1, \mathrm{~K} 2$ and $\mathrm{Km}$ were slightly decreased after the operation. Although we did not find significantly decreased $\mathrm{K}$ values, our results showed that corneal keratometry was stable at 1-year after the operation, which is absolutely valuable for keratoconus treatment.

We also found that the decrease in the corneal curvature was not consistent with the decrease in the corneal refractive power. Thus, the mechanism of this technology for refractive error correction may include not only corneal flattening but also other factors, such as remodeling of the corneal epithelium. Nonetheless, further studies are required.

In our study, the shape of most corneal cones changed after the operation, where keratometry of part of the corneal cone decreased, while some cone ranges even decreased. Keratometry of most parts was stable outside the corneal cone. This result was different from that of traditional or accelerated corneal cross-linking without a topography guide, which usually causes a decrease in keratometry both in and out of the corneal cone. In addition, the shape and range of the cone did not change. Regarding the mechanism of the technique, topography-guided corneal collagen cross-linking can select different irradiation energies applied to different parts of the cornea according to the corneal topography. A greater curvature of the cornea receives more UVA energy, and thus, more energy can be given to the cone area than to the peripheral parts so that the cone can be further flattened. Our results confirmed this theory to some extent, and we believe that topography guidance should play a certain role in this operation. Thus, this technique may give patients a more personalized treatment, selectively reduce the steep portion of the corneal curvature, smooth irregular corneas, and improve visual acuity.

Our results were a little different from those published case reports, which demonstrated more obvious improvement in vision and decreased corneal curvature. For example, in the case report of Kanellopoulos et al ${ }^{7}$, the $\mathrm{K}$ value of 4 eyes decreased by 2.3D and 1.44D at 1 week and 6 months post-operatively, respectively. We should note that our study was prospective and enrolled more patients, and thus, the scientificity and reliability should be better compared to the case report; however, we also found that the Kanellopoulos study used a UVA of $12 \mathrm{~J}$, while the average corneal curvature was 44.5D. In the present study we used much less UVA energy, which might affect the cross-linking. Better results might be obtained with a modified design in future studies.

In conclusion, we present a new technique that may be applied in keratoconus treatment for low refractive error correction; however, further studies and improvement are needed in topography-guided transepithelial accelerated corneal collagen cross-linking.

\section{References}


1. Wollensak G, Spoerl E, Seiler T. Riboflavin/ultraviolet-a-induced collagen crosslinking for the treatment of keratoconus. American journal of ophthalmology. May 2003;135(5):620-627.

2. Vinciguerra R, Romano MR, Camesasca Fl, et al. Corneal cross-linking as a treatment for keratoconus: four-year morphologic and clinical outcomes with respect to patient age. Ophthalmology. May 2013;120(5):908-916.

3. Kanellopoulos AJ. Long term results of a prospective randomized bilateral eye comparison trial of higher fluence, shorter duration ultraviolet A radiation, and riboflavin collagen cross linking for progressive keratoconus. Clinical ophthalmology. 2012;6:97-101.

4. Chow VW, Chan TC, Yu M, Wong VW, Jhanji V. One-year outcomes of conventional and accelerated collagen crosslinking in progressive keratoconus. Scientific reports. 2015;5:14425.

5. Wittig-Silva C, Chan E, Islam FM, Wu T, Whiting M, Snibson GR. A randomized, controlled trial of corneal collagen cross-linking in progressive keratoconus: three-year results. Ophthalmology. Apr 2014;121(4):812-821.

6. Roy AS, Dupps WJ, Jr. Patient-specific computational modeling of keratoconus progression and differential responses to collagen cross-linking. Investigative ophthalmology \& visual science. 2011;52(12):9174-9187.

7. Kanellopoulos AJ. Novel myopic refractive correction with transepithelial very high-fluence collagen cross-linking applied in a customized pattern: early clinical results of a feasibility study. Clinical ophthalmology. 2014;8:697-702.

8. Kanellopoulos AJ, Dupps WJ, Seven I, Asimellis G. Toric topographically customized transepithelial, pulsed, very high-fluence, higher energy and higher riboflavin concentration collagen cross-linking in keratoconus. Case reports in ophthalmology. May 2014;5(2):172-180.

9. Sun L, Li M, Zhang X, et al. Transepithelial accelerated corneal collagen cross-linking with higher oxygen availability for keratoconus: 1-year results. International ophthalmology. Dec 2018;38(6):2509-2517.

\section{Table 1}

Table 1 Ocular examination results at each follow-up point. 


\begin{tabular}{|c|c|c|c|c|c|c|}
\hline & pre & $1 \mathrm{w}$ po & $1 \mathrm{~m}$ po & $3 \mathrm{~m}$ po & $6 \mathrm{~m}$ po & 1у ро \\
\hline $\begin{array}{l}\text { UDVA(logMAR) } \\
(\text { mean } \pm S D)\end{array}$ & $0.67 \pm 0.09$ & $0.48 \pm 0.07$ & $0.48 \pm 0.07$ & $0.49 \pm 0.07$ & $0.60 \pm 0.07$ & $0.52 \pm 0.08$ \\
\hline$P$ & & 0.40 & 0.44 & 1 & 1 & 1 \\
\hline $\begin{array}{l}\text { CDVA(logMAR) } \\
(\text { mean } \pm S D)\end{array}$ & $0.09 \pm 0.04$ & $0.04 \pm 0.03$ & $0.03 \pm 0.02$ & $0.02 \pm 0.03$ & $0.05 \pm 0.04$ & $0.05 \pm 0.04$ \\
\hline$P$ & & 0.52 & 0.33 & 0.37 & 1 & 1 \\
\hline $\begin{array}{l}\mathrm{SE}(\mathrm{D}) \\
(\text { mean } \pm S D)\end{array}$ & $-3.62 \pm 0.39$ & $-3.30 \pm 0.35$ & $-3.23 \pm 0.32$ & $-3.07 \pm 0.32$ & $-3.01 \pm 0.34$ & $-2.90 \pm 0.34$ \\
\hline$P$ & & 0.83 & 0.55 & 0.45 & 0.31 & 0.17 \\
\hline $\begin{array}{l}\text { Steep K(D) } \\
\text { (mean } \pm S D)\end{array}$ & $46.44 \pm 40.70$ & $46.13 \pm 0.65$ & $46.29 \pm 0.66$ & $46.19 \pm 0.65$ & $46.17 \pm 0.64$ & $46.15 \pm 0.60$ \\
\hline$P$ & & 0.12 & 0.09 & 0.10 & 0.10 & 0.14 \\
\hline $\begin{array}{l}\text { Flat K(D) } \\
(\text { mean } \pm S D)\end{array}$ & $44.40 \pm 0.44$ & $44.14 \pm 0.41$ & $44.28 \pm 0.44$ & $44.24 \pm 0.42$ & $44.19 \pm 0.41$ & $44.28 \pm 0.44$ \\
\hline$P$ & & 0.13 & 1 & 1 & 0.61 & 1 \\
\hline $\begin{array}{l}\mathrm{K} \max (\mathrm{D}) \\
(\text { mean } \pm S D)\end{array}$ & $49.13 \pm 1.22$ & $48.98 \pm 1.22$ & $49.11 \pm 1.31$ & $48.76 \pm 1.09$ & $48.77 \pm 1.14$ & $48.82 \pm 1.16$ \\
\hline$P$ & & 1 & 1 & 0.95 & 0.35 & 0.77 \\
\hline $\begin{array}{l}\text { TCT }(\mu \mathrm{m}) \\
(\text { mean } \pm S D)\end{array}$ & $497.25 \pm 5.69$ & $485.69 \pm 6.62$ & $489.38 \pm 6.29$ & $493.94 \pm 6.67$ & $492.25 \pm 7.07$ & $492.00 \pm 6.86$ \\
\hline$P$ & & 0.01 & 0.19 & 1 & 1 & 0.84 \\
\hline $\begin{array}{l}\text { ECD } \\
(\text { mean } \pm S D)\end{array}$ & $3095.01 \pm 98.85$ & $3016.15 \pm 122.88$ & $3047.27 \pm 104.38$ & $3255.69 \pm 109.20$ & $3307.11 \pm 116.27$ & $3269.93 \pm 126.46$ \\
\hline$P$ & & 1 & 1 & 1 & 1 & 1 \\
\hline
\end{tabular}

UDVA uncorrected distant visual acuity; CDVA corrected distant visual acuity; SE spherical equivalent; TCT thinnest corneal thick; ECD endothelial cell density; P value were compared with the preoperative and postoperative follow-up.

\section{Figures}
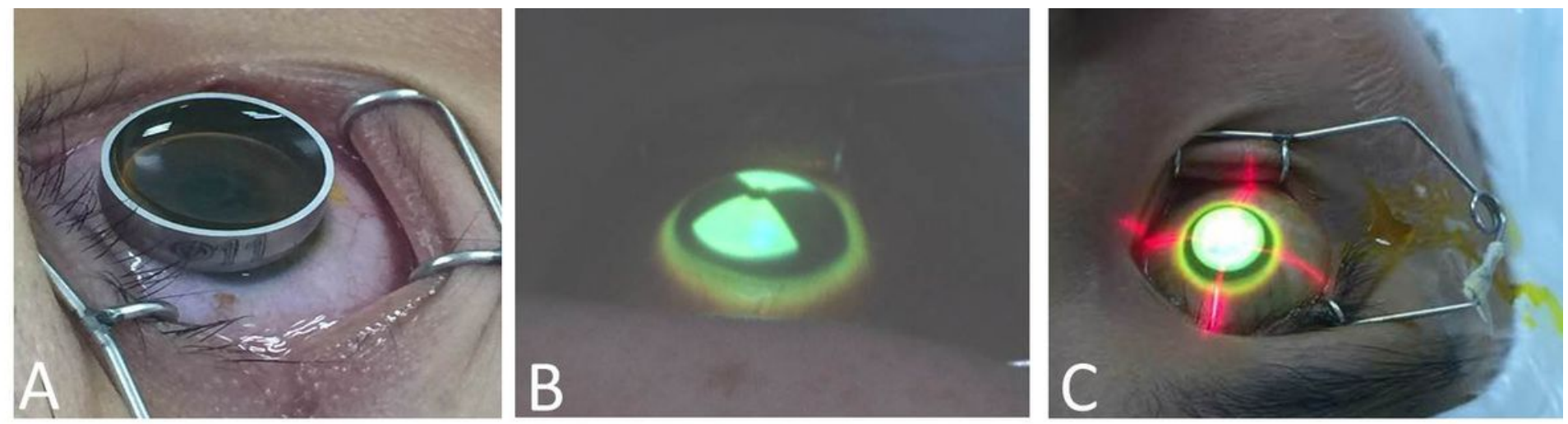


\section{Figure 1}

The operation process. (A) Application of riboflavin. (B) According to the corneal topography, UVA was applied in a sector shape. (C) According to the corneal topography, UVA was applied in a circular shape.

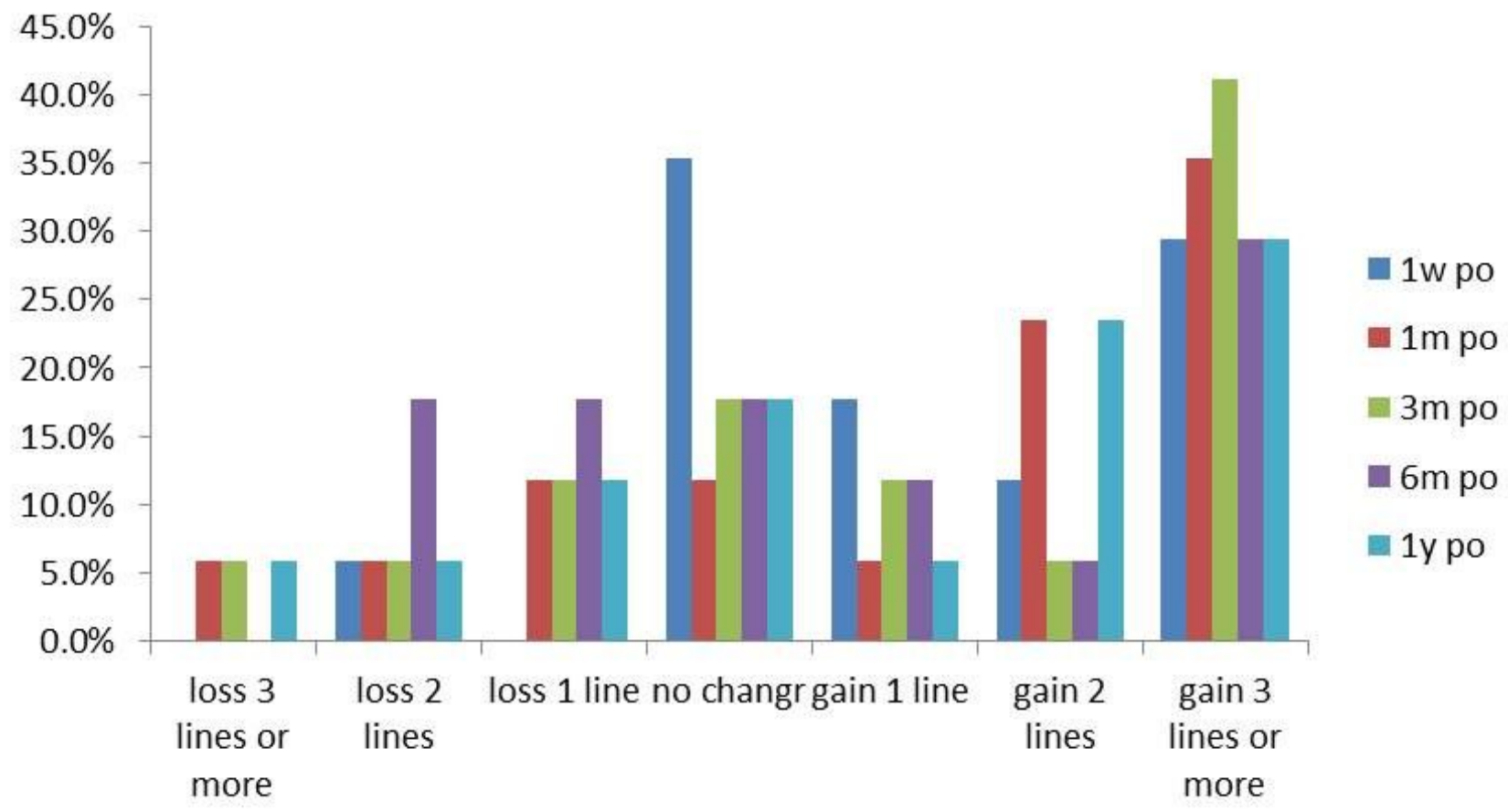

\section{Figure 2}

The UCVA of most patients was higher than the pre-operative value, and the UCVA of nearly $1 / 3$ of the patients was enhanced by at least 3 lines at each follow-up visit. 


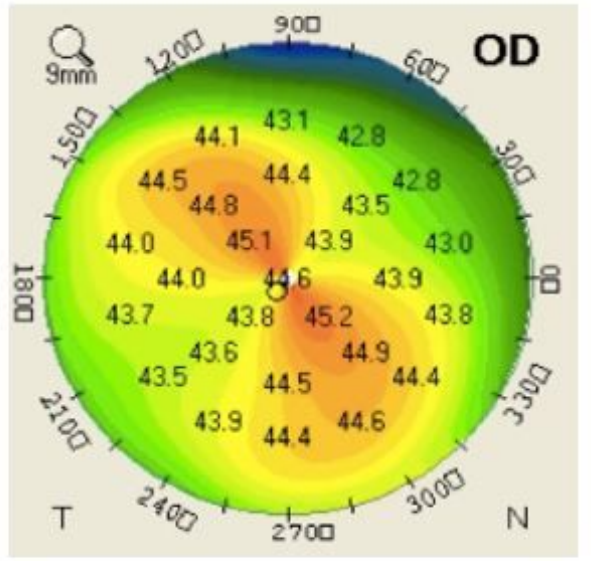

Fig.3A Anterior surface of cornea before operation

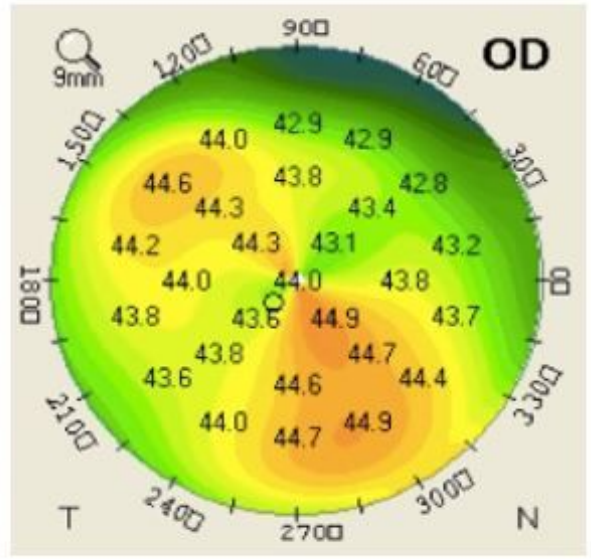

Fig.3C Further changes in the shape of the cone at 3 month after operation

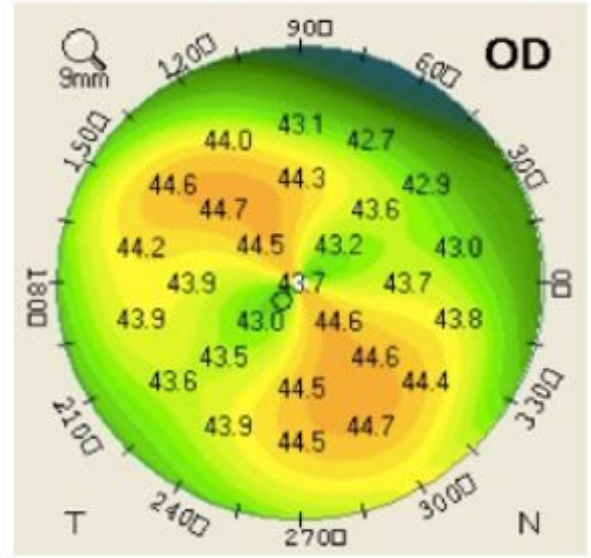

Fig.3B Corneal curvature decreased and cone shape changed at 1 week after operation

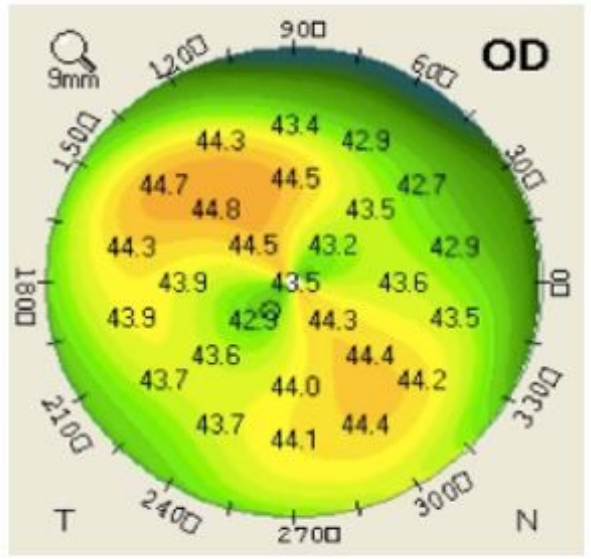

Fig.3D The curvature of the cone decreased and its shape changed continually at 1 year after operation

Figure 3

The shape of corneal cone changed during the whole follow-up period after the operation. 